\section{Comet tail artifact on ultrasonography: is it a reliable finding of benign gallbladder diseases?}

\author{
Sung Hoon Oh, Hyun Young Han, Hee Jin Kim \\ Department of Radiology, Eulji University Hospital, Daejeon, Korea
}

Purpose: The aim of this study was to evaluate whether the comet tail artifact on ultrasonography can be used to reliably diagnose benign gallbladder diseases.

Methods: This retrospective study reviewed the clinical findings, imaging findings, preoperative ultrasonographic diagnoses, and pathological diagnoses of 150 patients with comet tail artifacts who underwent laparoscopic cholecystectomy with pathologic confirmation. The extent of the involved lesion was classified as localized or diffuse, depending on the degree of involvement and the anatomical section of the gallbladder that was involved. This study evaluated the differences in clinical and imaging findings among pathologic diagnoses.

Results: All gallbladder lesions exhibiting the comet tail artifact on ultrasound examination were confirmed as benign gallbladder diseases after cholecystectomy, including 71 cases of adenomyomatosis (47.3\%), 74 cases of chronic cholecystitis (49.3\%), two cases of xanthogranulomatous cholecystitis (1.3\%), and three cases of cholesterolosis (2.0\%); there were two cases of coexistent chronic cholecystitis and low-grade dysplasia. There were no statistically significant differences in any of the clinical and ultrasonographic findings, with the exception of gallstones $(P=0.007)$, among the four diseases. There were no significant differences in the average length, thickness, or number of comet tail artifacts among the four diagnoses. No malignancies were detected in any of the 150 thickened gallbladder lesions.

Conclusion: The ultrasonographic finding of the comet tail artifact in patients with thickened gallbladder lesions is associated with the presence of benign gallbladder diseases, and can be considered a reliable sign of benign gallbladder disease.

Keywords: Gallbladder; Ultrasonography; Artifact

\section{Introduction}

Ultrasonography is an essential imaging modality in diagnosing patients with gallbladder disease. If an ultrasound examination reveals a thickened gallbladder wall, malignant and benign gallbladder lesions, such as cholecystitis, benign cholesterolosis, and adenomyomatosis, among others, should be distinguished $[1,2]$. It is important to distinguish between benign and malignant gallbladder lesions because the treatment methods and prognoses are different for each.

Imaging features including invasion of the surrounding tissue, growth of large-sized polypoid

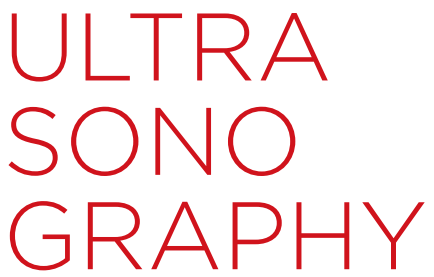

ORIGINAL ARTICLE

https://doi.org/10.14366/usg. 18029 pISSN: 2288-5919 • elSSN: 2288-5943

Ultrasonography 2019;38:221-230

Received: May 21, 2018

Revised: October 2, 2018

Accepted: October 3, 2018

Correspondence to:

Hyun Young Han, MD, PhD, Department of Radiology, Eulji University Hospital, 95 Dunsanseo-ro, Seo-gu, Daejeon 35233, Korea

Tel. $+82-42-611-3563$

Fax. +82-42-611-3590

E-mail: hanhy@eulji.ac.kr

This is an Open Access article distributed under the terms of the Creative Commons Attribution NonCommercial License (http://creativecommons.org/ licenses/by-nc/4.0//) which permits unrestricted noncommercial use, distribution, and reproduction in any medium, provided the original work is properly cited.

Copyright (C) 2019 Korean Society of Ultrasound in Medicine (KSUM)

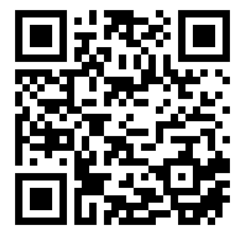

How to cite this article:

Oh SH, Han HY, Kim HJ. Comet tail artifact on ultrasonography: is it a reliable finding of benign gallbladder diseases? Ultrasonography. 2019 Jul;38(3):221-230. 
lesions, and restriction on diffusion-weighted magnetic resonance imaging (MRI) are well-known features of malignant gallbladder lesions [3-5]. Many attempts have been made to distinguish between benign and malignant gallbladder lesions using various imaging modalities, such as contrast-enhanced computed tomography and MRI [6-10]. However, gallbladder cancer exhibits a variety of imaging features [3]. Occasionally, it is difficult to distinguish between benign and malignant gallbladder lesions in cases that do not exhibit specific malignant features, such as minimal wall thickening and comparable enhancement to that of the adjacent normal wall of the gallbladder.

The comet tail artifact is a well-known useful reverberation artifact on sonograms [11]. It is often accompanied by a thickened gallbladder wall lesion, such as gallbladder adenomyomatosis. The presence or absence of the comet tail artifact depends on the configuration of the gallbladder lesions. However, no research has investigated whether the comet tail artifact appears in malignant gallbladder lesions. The aim of this study was to evaluate whether the comet tail artifact on ultrasonography can be used as a reliable finding in the diagnosis of benign gallbladder diseases.

\section{Materials and Methods}

The Institutional Review Board approved this retrospective study and waived the requirement for patients to provide informed consent.

\section{Patient Selection}

This study reviewed 19,361 abdominal ultrasound examinations performed at the authors' institution between January 2012 and December 2016. In total, 296 cases exhibited a comet tail artifact at a gallbladder lesion on ultrasound examination. Among these, 64 cases were excluded because they involved patients who underwent repeated examinations, while 82 patients were excluded because they did not undergo surgery or were lost to follow-up. A total of 150 patients who underwent cholecystectomy were included in the final data analysis (Fig. 1).

The following information was collected from the patients' electronic medical records: age, sex, presence of accompanying biliary colic symptoms, preoperative ultrasonographic diagnosis, surgical indication of cholecystectomy, final pathological diagnosis, and time interval between the ultrasound examination and cholecystectomy. In some cases, there were at least two pathologic diagnoses. In these cases, the primary finding was used to determine the final pathologic diagnosis.

\section{Gallbladder Ultrasonography}

Gallbladder ultrasonography was performed using an ultrasonography unit equipped with a 1-5-MHz convex array transducer (iU22, Philips Medical System, Bothell, WA, USA). Ultrasound images of the gallbladder were acquired using the grayscale B-mode imaging technique and harmonic technique, with or without the zoom function. The examinations were performed with the patients in a fasting state and in either the supine or left lateral decubitus position. Two expert radiologists with more than 20 years of abdominal imaging experience performed the gallbladder ultrasonography.

\section{Ultrasonographic Analysis}

The comet tail artifact was defined as the presence of both a bright hyperechoic focus at the gallbladder lesion and an inverted triangular acoustic enhancement posterior to the hyperechoic focus, which exhibited a gradual decrease in strength and thickness (Fig. 2).

\section{9,361 Routine abdomen ultrasonography} in 2012 January-2016 December

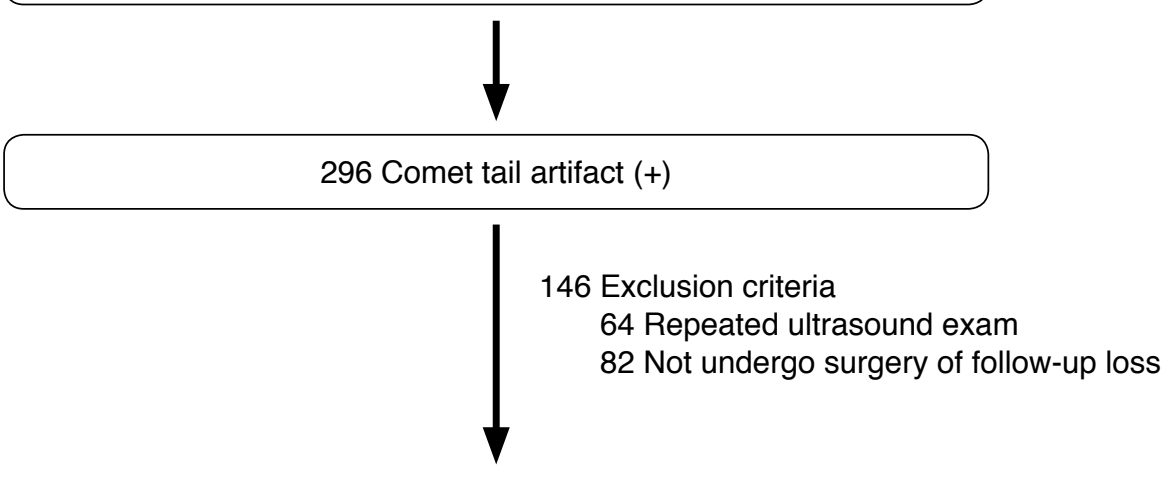

150 Finally included patients with cholecystectomy
Fig. 1. Inclusion criteria for patient selection. This flow chart shows the exclusion criteria and the number of patients excluded, and the number of patients finally included. 
The maximal length and thickness of the comet tail artifact was measured in millimeters, and the values were rounded to the first decimal place. The number of comet tail artifacts was counted in the image that was most frequently observed.

The maximum thickness of the gallbladder wall lesion was measured through the inner to outer margin at the greatest thickness of the lesion, in either intercostal or longitudinal gallbladder ultrasound images, and expressed in millimeters with rounding to the nearest millimeter. In this study, a gallbladder lesion with a width greater than $3 \mathrm{~mm}$ was considered to be thickened. In all ultrasound images, irregularity of the lesions' margin, loss of the wall layer, presence of intralesional cysts, and echogenic foci were examined; in addition, the presence of an accompanying gallstone, invasion of adjacent organs, lymph node enlargement, and collapse of the gallbladder lumen were assessed.

It has been suggested that the extent of involvement is an important factor influencing the assessment of whether a gallbladder lesion constitutes a malignant lesion. Thus, it was necessary to investigate whether there was a difference in the proportion of malignant lesions according to their extent of involvement. Because no standard method exists to distinguish the extent of gallbladder lesion involvement, the lesions were divided into two types according to their extent of involvement on anatomical sections.
The gallbladder is commonly divided into fundus, body, and neck sections; thus, a lesion was classified as localized if the lesion was present in one section, and diffuse if it was present in more than one section contiguously (Fig. 3).

\section{Statistical Analysis}

The Kruskal-Wallis test was used to compare the average values of patient age and lesion thickness, as well as the length, thickness, and number of comet tail artifacts among the final pathologic diagnoses. The Fisher exact test was used to compare the frequencies of patients' sex and the presence of accompanying biliary colic symptoms, lesion wall irregularity, loss of the wall layer, intralesional cyst, echogenic foci, a collapsed gallbladder lumen, accompanying gallstone, enlarged lymph node, and invasion of surrounding tissue among the pathologic diagnoses of gallbladder lesions. If statistical significance was observed for a variable, the Wilcoxon rank-sum test was used to determine whether a statistically significant difference was present in the average values within each diagnosis, while the Fisher exact test was used to determine whether a statistically significant difference was present in the proportions of diagnoses. All statistical analyses were performed using R software ver. 3.5.1 (https://cran.r-project.org), an open-source software distributed under the GNU General Public License. Additional packages for R

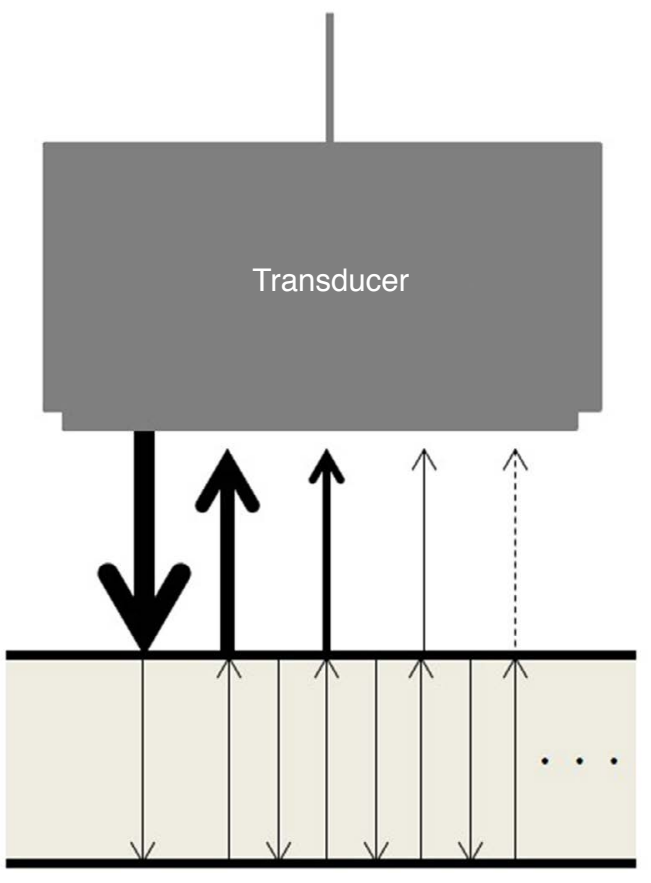

A

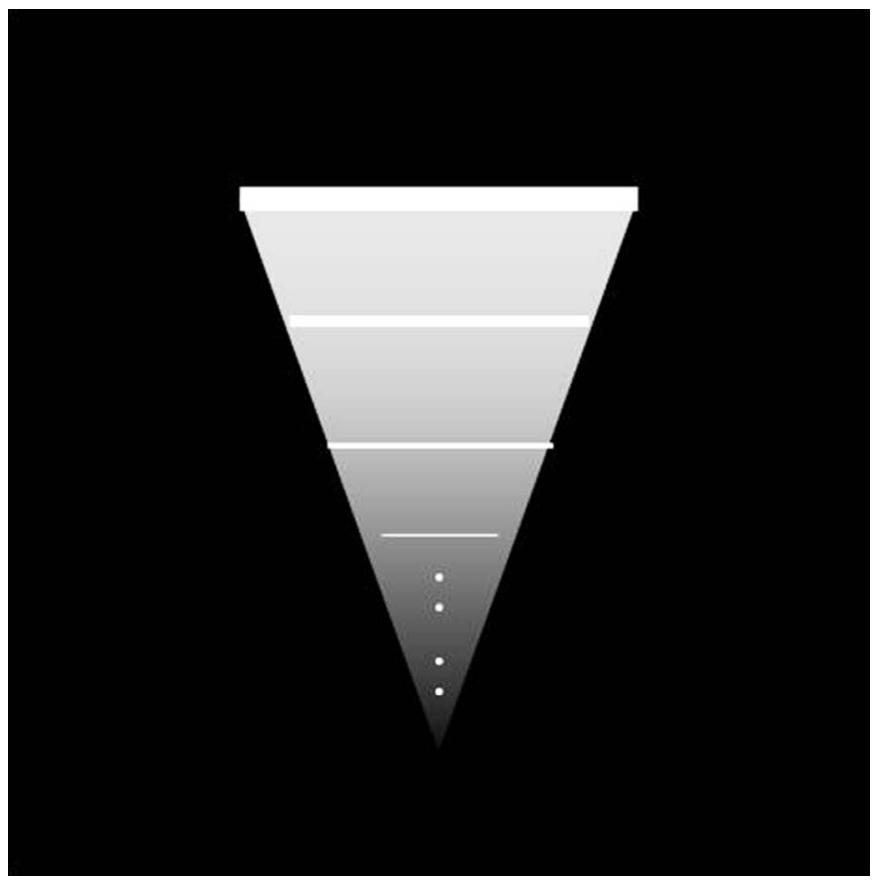

B

Fig. 2. Schematic representation of the comet tail artifact.

A. The ultrasonic beam is repeatedly reflecting on the shallow and deep sides of matter, and the reflected beams are returning to the probe again. B. This figure shows a sonogram of a triangular hyperechoic lesion made up of repeatedly reflected beams coming into probe. 
were obtained from mirror sites of the Comprehensive R Archive Network (CRAN) (https://github.com/cardiomoon/moonBook, https://userfriendlyscience.com/download). P-values of $<0.05$ were considered to indicate statistical significance.

\section{Results}

Of the 150 patients included in the final data analysis, the respective numbers of male and female patients were 85 (56.7\%) and $65(43.3 \%)$. The mean age was $44.1 \pm 11.0$ years (range, 21 to 84 years). Tables 1 and 2 summarize the patients' preoperative clinical and ultrasonographic diagnoses before cholecystectomy. The preoperative clinical diagnosis was made after ultrasonography, and corresponded directly to the surgical indication. The included preoperative ultrasonographic diagnoses were as follows: acute cholecystitis, chronic cholecystitis, adenomyomatosis, cholesterolosis, benign polyp, xanthogranulomatous cholecystitis, and gallbladder carcinoma (Table 2). Five patients were diagnosed with gallbladder carcinoma on preoperative ultrasonography (3.3\%) (Fig. 4). In the five patients with suspected malignant lesions, one was a localized lesion with wall thickness of $5 \mathrm{~mm}$, a maximal diameter of 12 $\mathrm{mm}$, and marginal irregularity; loss of the wall layer was observed on ultrasonography, and the lesion was diagnosed as chronic cholecystitis after cholecystectomy. Another patient exhibited a
Table 1. The number of patients according to clinical indications of cholecystectomy

\begin{tabular}{lc}
\hline \multicolumn{1}{c}{ Indication } & No. \\
\hline Acute cholecystitis & 30 \\
Adenomyomatosis with gallstone & 45 \\
Adenomyomatosis without gallstone & 19 \\
Biliary colic symptom with gallstone & 46 \\
Biliary colic symptom without gallstone & 4 \\
Chronic cholecystitis & 1 \\
Suspected gallbladder carcinoma & 5 \\
\hline
\end{tabular}

Table 2. The number of patients according to the preoperative ultrasonographic diagnosis

\begin{tabular}{lc}
\hline \multicolumn{1}{c}{ Diagnosis } & No. (\%) \\
\hline Adenomyomatosis & $95(63.3)$ \\
Adenomyomatosis with acute cholecystitis & $24(16.0)$ \\
Adenomyomatosis with cholesterolosis & $8(5.3)$ \\
Acute cholecystitis & $6(4.0)$ \\
Benign polyp & $6(4.0)$ \\
Chronic cholecystitis with cholesterolosis & $3(2.0)$ \\
Chronic cholecystitis & $1(0.7)$ \\
Xanthogranulomatous cholecystitis & $2(1.3)$ \\
Gallbladder carcinoma & $5(3.3)$ \\
\hline
\end{tabular}

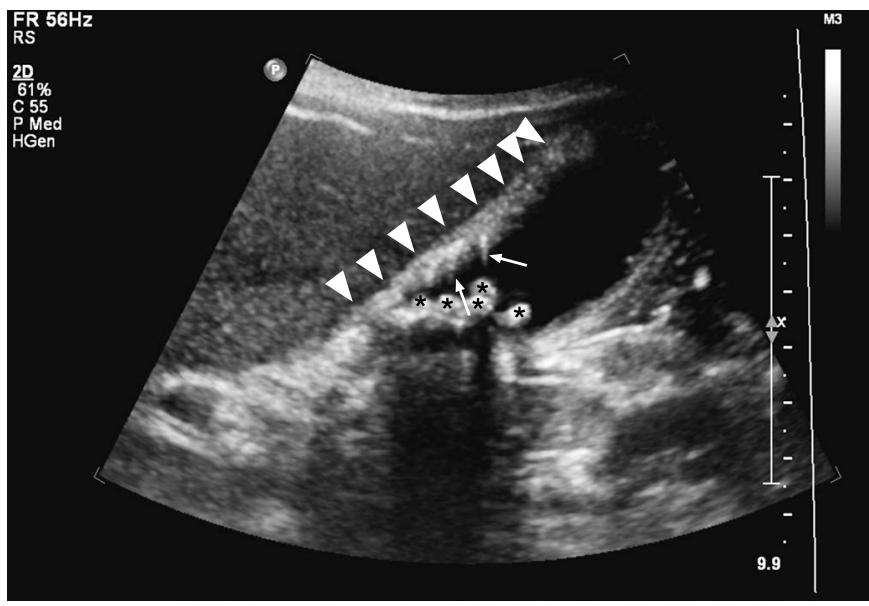

B

Fig. 3. Representative cases of the comet tail artifact.

A. A 34-year-old man with incidentally detected localized type gallbladder wall thickening on ultrasonography, pathologically confirmed as adenomyomatosis. Transabdominal ultrasonography shows localized wall thickening (arrowheads) at the fundus of the gallbladder with comet tail artifacts (arrows) (wall thickness: approximately $6 \mathrm{~mm}$ ). The presence of sludge is observed in the lumen of the gallbladder (asterisk). B. A 37-year-old man with incidentally detected diffuse type gallbladder wall thickening on ultrasonography, pathologically confirmed as chronic cholecystitis. Transabdominal ultrasonography shows diffuse, irregular wall thickening (arrowheads) at the fundus, body, and neck of the gallbladder with multiple comet tail artifacts (arrows) (wall thickness: approximately $5 \mathrm{~mm}$ ). Note multiple gallstones in the lumen of the gallbladder (asterisks). 
diffuse lesion with a wall thickness of $9 \mathrm{~mm}$, an accompanying irregular margin, and loss of the wall layer; this lesion was diagnosed as adenomyomatosis after the gallbladder operation. Two patients had lesions that were suspected to be malignant; these were diffuse, relatively thick (approximately $6 \mathrm{~mm}$ ), and newly appeared lesions that demonstrated a loss of the wall layer. These patients were diagnosed with chronic cholecystitis and cholesterolosis after cholecystectomy, respectively. In the other patient, the lesion was diffuse with a wall thickness of $6 \mathrm{~mm}$, an accompanying irregular margin, and loss of the wall layer; it was diagnosed as adenomyomatosis after cholecystectomy.

Of the 150 patients, 74 (49.3\%) had accompanying biliary colic symptoms. The average maximum thickness of the gallbladder lesion on ultrasonographic imaging was $5.7 \mathrm{~mm}$ (range, 3 to $14 \mathrm{~mm}$ ). The number of diffuse lesions was 91 (60.7\%), and the number of localized lesions was 59 (39.3\%). Twenty-nine patients (19.3\%) exhibited an irregular margin and 96 (64.0\%) showed a loss of the wall layer. Four patients (2.7\%) demonstrated an intralesional cystic lesion and 22 (14.7\%) exhibited intralesional echogenic foci. The number of lesions with accompanying gallstones was 120 (80.0\%). There were nine patients (6.0\%) with a collapsed gallbladder lumen. The average values of the maximum length and thickness of the comet tail artifact were $7.6 \mathrm{~mm}$ and $3.2 \mathrm{~mm}$, respectively; the average number of comet tail artifacts was 3.0. No patients showed involvement of an adjacent organ or an accompanying enlarged lymph node.

The final pathological diagnoses after cholecystectomy of the 150 patients in this study are shown in Fig. 5. All 150 thickened gallbladder lesions yielded findings of benign pathology on cholecystectomy, including 71 cases (47.3\%) of adenomyomatosis with chronic inflammation; 74 cases $(49.3 \%)$ of chronic cholecystitis without adenomyomatosis; two cases $(1.3 \%)$ of xanthogranulomatous cholecystitis; and three cases $(2.0 \%)$ of cholesterolosis only. Of 74 chronic cholecystitis lesions, two were accompanied by low-grade dysplasia: one patient was a 32-yearold woman with a localized, thickened gallbladder lesion (maximum thickness, $4 \mathrm{~mm}$ ) and a 14-day interval between ultrasonography and cholecystectomy. The other patient was a 35-year-old man with a diffuse, thickened gallbladder lesion (maximum thickness, $4 \mathrm{~mm}$ ) and a 7-day interval between ultrasonography and cholecystectomy (Fig. 6). No malignancy was present in any of the 150 thickened gallbladder lesions.

Table 3 summarizes the clinical and imaging findings based on the final pathologic diagnoses, including chronic cholecystitis, adenomyomatosis, cholesterolosis, and xanthogranulomatous cholecystitis. There were no statistically significant differences in the mean age $(P=0.145)$, sex ratio $(P=0.667)$, or the presence of biliary colic symptoms $(P=0.163)$ among the four diseases. With

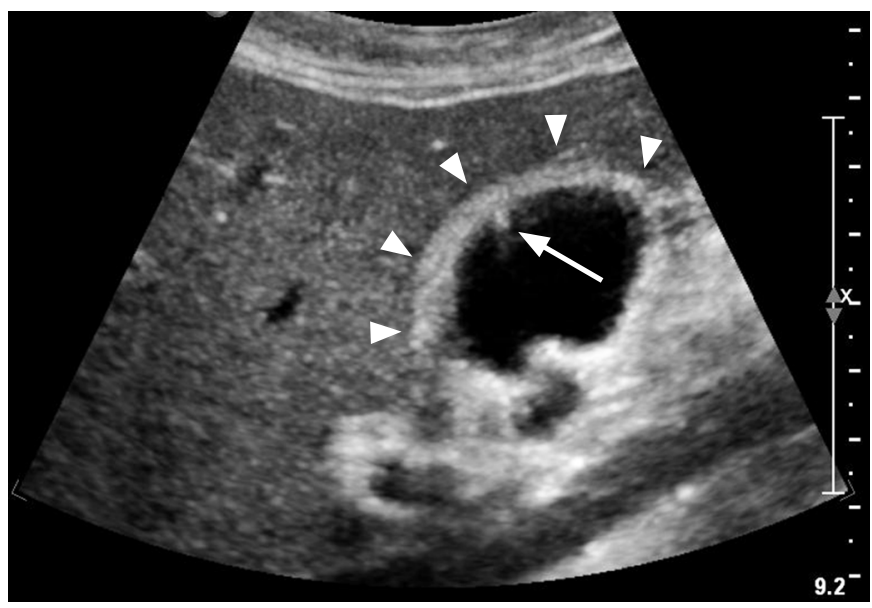

B

Fig. 4. Two cases suspected malignant lesions on preoperative ultrasonography.

A. A 40-year-old woman with incidentally detected diffuse gallbladder wall thickening on ultrasonography, pathologically confirmed as adenomyomatosis. Transabdominal ultrasonography shows diffuse, irregular wall thickening (arrowheads) at the fundus and body of the gallbladder with comet tail artifacts (arrows) (wall thickness: approximately $9 \mathrm{~mm}$ ). The lesion shows loss of wall layer. This lesion is not accompanied by gallstones. B. A 45-year-old man with newly detected diffuse gallbladder wall thickening on ultrasonography, pathologically confirmed as chronic cholecystitis. Transabdominal ultrasonography shows diffuse wall thickening (arrowheads) of the gallbladder with comet tail artifact (arrow) (wall thickness: approximately $6 \mathrm{~mm}$ ). The lesion shows loss of wall layer. This lesion has a relatively smooth margin and is not accompanied by gallstones. 


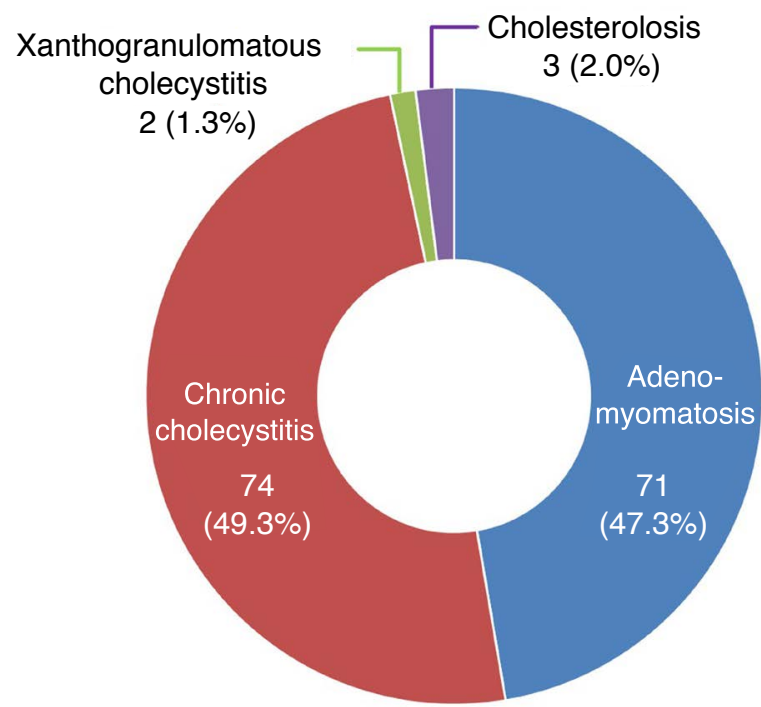

Fig. 5. Schematic drawing of the pathological diagnosis of patients included in the study $(n=150)$. All 150 thickened gallbladder lesions yielded the finding of benign pathology on cholecystectomy, including $71(47.3 \%)$ adenomyomatosis with chronic inflammation; 74 (49.3\%) chronic cholecystitis without adenomyomatosis; two (1.3\%) xanthogranulomatous cholecystitis; and three $(2.0 \%)$ cholesterolosis only.

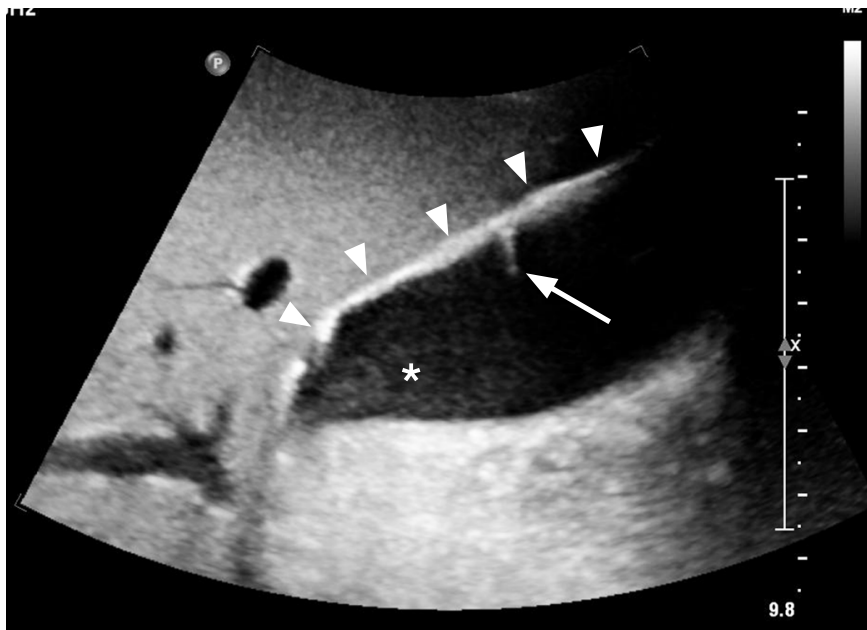

Fig. 6. A case of low-grade dysplasia detected on a final pathologic exam. A 35-year-old man with incidentally detected diffuse gallbladder wall thickening on ultrasonography, pathologically confirmed as chronic cholecystitis with low grade dysplasia. Transabdominal ultrasonography shows mild diffuse wall thickening (arrowheads) at the body and neck of the gallbladder with comet tail artifact (arrow) (wall thickness: approximately 4 $\mathrm{mm}$ ). The presence of sludge is observed in the lumen of gallbladder (asterisk). This lesion did not demonstrate intramural cystic lesion, irregular wall, or invasion to surrounding tissues.

Table 3. Clinical and ultrasound characteristics of included patients with a thickened gallbladder wall

\begin{tabular}{|c|c|c|c|c|c|c|}
\hline & $\begin{array}{l}\text { Chronic chole- } \\
\text { cystitis }(n=74)\end{array}$ & $\begin{array}{c}\text { Adenomyo- } \\
\text { matosis }(n=71)\end{array}$ & $\begin{array}{c}\text { Cholesterolosis } \\
(n=3)\end{array}$ & $\begin{array}{c}\text { Xanthogranulomatous } \\
\text { cholecystitis }(n=2)\end{array}$ & Total $(n=150)$ & P-value \\
\hline Age (yr) & $44.4 \pm 10.9$ & $43.3 \pm 10.6$ & $44.3 \pm 12.0$ & $67.0 \pm 7.0$ & $44.1 \pm 11.0$ & 0.145 \\
\hline Male sex & $42(56.8)$ & $40(56.3)$ & $1(33.3)$ & $2(100)$ & $85(56.7)$ & 0.667 \\
\hline Female & $32(43.2)$ & $31(43.7)$ & $2(66.7)$ & 0 & $65(43.3)$ & \\
\hline Biliary colic symptom & $41(55.4)$ & $30(42.3)$ & $1(33.3)$ & $2(100)$ & $74(49.3)$ & 0.163 \\
\hline Maximum wall thickness (mm) & $5.5 \pm 1.9$ & $5.9 \pm 2.4$ & $5.7 \pm 0.5$ & $8.5 \pm 1.5$ & $5.7 \pm 2.2$ & 0.227 \\
\hline Localized type & $31(41.9)$ & $27(38.0)$ & 0 & $1(50)$ & $59(39.3)$ & 0.338 \\
\hline Diffuse type & $43(58.1)$ & $44(62.0)$ & $3(100)$ & $1(50)$ & $91(60.7)$ & \\
\hline Irregularity & $13(17.6)$ & $15(21.1)$ & 0 & $1(50)$ & $29(19.3)$ & 0.507 \\
\hline Loss of layer & $46(62.2)$ & $47(66.2)$ & $2(66.7)$ & $1(50)$ & $96(64.0)$ & 0.944 \\
\hline Cystic lesion & $2(2.7)$ & $2(2.8)$ & 0 & 0 & $4(2.7)$ & $>0.990$ \\
\hline Echogenic foci & $11(14.9)$ & $11(15.5)$ & 0 & 0 & $22(14.7)$ & $>0.990$ \\
\hline Gallstone & $66(89.2)$ & $51(71.8)$ & $1(33.3)$ & $2(100)$ & $120(80.0)$ & $0.007^{\mathrm{a})}$ \\
\hline Collapsed lumen & $2(2.7)$ & $7(9.9)$ & 0 & 0 & $9(6.0)$ & 0.578 \\
\hline Maximum length of the CTA (mm) & $7.4 \pm 3.0$ & $7.8 \pm 3.9$ & $7.0 \pm 4.5$ & $8.0 \pm 2.0$ & $7.6 \pm 3.5$ & 0.940 \\
\hline Maximum thickness of the CTA (mm) & $3.2 \pm 1.1$ & $3.3 \pm 1.2$ & $2.7 \pm 0.9$ & $2.5 \pm 0.5$ & $3.2 \pm 1.1$ & 0.576 \\
\hline No. of CTAs & $3.0 \pm 1.5$ & $3.0 \pm 2.0$ & $5.7 \pm 5.2$ & $2.0 \pm 1.0$ & $3.0 \pm 1.9$ & 0.670 \\
\hline
\end{tabular}

Values are presented as mean \pm standard deviation or number (\%).

There were no cases involving adjacent organs or accompanying enlarged lymph nodes.

CTA, comet tail artifact.

${ }^{\text {a) }}$ Statistically significant P-value. 
Table 4. P-values from the Fisher exact test comparing the proportions of accompanying gallstones in each diagnosis

\begin{tabular}{lcccc}
\hline & Chronic cholecystitis & Adenomyomatosis & Cholesterolosis & Xanthogranulomatous cholecystitis \\
\hline Chronic cholecystitis & - & $0.011^{\mathrm{a})}$ & $0.043^{\mathrm{a})}$ & 1 \\
Adenomyomatosis & $0.011^{\mathrm{a})}$ & - & 0.209 & 1 \\
Cholesterolosis & $0.043^{\mathrm{a})}$ & 0.209 & - & 0.400 \\
Xanthogranulomatous cholecystitis & 1 & 1 & 0.400 & - \\
\hline
\end{tabular}

Statistical analysis was performed using the Fisher exact test to compare the ratios between groups, and P-values were assessed.

a) Statistically significant P-value.

regard to ultrasonographic findings, the proportion of lesions with accompanying gallstones was $89.2 \%$ of patients with chronic cholecystitis, $71.8 \%$ of those with adenomyomatosis, $33.3 \%$ of those with cholesterolosis, and $100 \%$ of those with xanthogranulomatous cholecystitis, respectively. There was a statistically significant difference in the percentage of patients with accompanying gallstones among the four diseases $(P=0.007)$. The rate of accompanying gallstones in patients with chronic cholecystitis was significantly higher than among patients with adenomyomatosis $(P=0.011)$ or cholesterolosis $(P=0.043)$. There were no significant differences in the proportion of accompanying gallstones among the other diseases (Table 4). Likewise, there were no significant differences in the average value or percentage of other ultrasonographic findings among the four diagnoses.

\section{Discussion}

Ultrasonography images are created by a strong reflected beam that is first reflected from the surfaces of reflectors. However, if the ultrasound beam is reflected repeatedly between the front and back surfaces of the reflector, multiple reflected beams appear in the image, as if multiple reflectors are present in the posterior row. When the process is repeated, a smaller amplitude and the appearance of an inverted triangular shape are attained; this pattern is known as the comet tail or V-shaped artifact [11]. Comet tail artifacts are known to occur at interfaces where areas of different acoustic impedance are found; for example, at the valve prosthesis on echocardiography, or in areas of abdominal extraluminal free gas on abdominal ultrasonography $[12,13]$.

In addition, the artifact is often present in adenomyomatosis of the gallbladder. Comet tail artifacts are the result of cholesterol crystals deposited in Rokitansky-Aschoff sinuses (RASs), which is a consequence of increased endoluminal pressure $[14,15]$. However, RASs are also present in cases of chronic cholecystitis $[16,17]$. Xanthogranulomatous cholecystitis is caused by extravasation of bile into the gallbladder wall through ruptured RASs; hence, this condition is considered to be rich in RASs [16-18]. Most cases with chronic cholecystitis, and approximately $80 \%$ of cases with xanthogranulomatous cholecystitis, show accompanying cholelithiasis $[18,19]$. Despite a lack of studies regarding the component ratio of cholesterol and pigmented stone in patients with gallbladder disease, one study reported that approximately $90 \%$ of cases of chronic cholecystitis and approximately $97 \%$ of cases of xanthogranulomatous cholecystitis had accompanying cholesterol or mixed stones [20]. Cholesterol crystals are a precursor of cholesterol stones, created and grown through cholesterol supersaturation and nucleation in the gallbladder $[21,22]$. Thus, patients with cholesterol stones show large amounts of cholesterol crystals. In summary, comet tail artifacts are caused by an accumulation of cholesterol crystals in RASs and can be seen in cases of both chronic cholecystitis and xanthogranulomatous cholecystitis, as well as in cases of adenomyomatosis.

In the present study, gallbladder lesions showing comet tail artifacts were diagnosed as chronic cholecystitis, adenomyomatosis, cholesterolosis, and xanthogranulomatous cholecystitis, and there was no difference in the proportion of each diagnosis, even when groups were divided according to the extent of the involved lesion. Thus, except for cholesterolosis, gallbladder diseases with comet tail artifacts are consistent with the diseases in which RASs exist. Cholesterolosis or cholesterol polyp is caused by the accumulation of cholesterol-rich macrophages in the lamina propria of the gallbladder. No studies have confirmed a relationship between cholesterolosis and RAS presence [23]. However, results from our study indicated a significantly lower rate of gallstone involvement in cases of cholesterolosis (33.3\%) than in cases of chronic cholecystitis (89.2\%). Although not statistically significant, the rate of accompanying gallstones in cholesterolosis was lower than in adenomyomatosis $(71.8 \%)$ or xanthogranulomatous cholecystitis $(100 \%)$. Notably, there was a small number of patients with cholesterolosis and xanthogranulomatous cholecystitis, which limited the statistical robustness of the results; the association between cholesterolosis and gallstones remains controversial in the literature [23]. This study could not find any association between cholesterolosis and RASs, or between cholesterolosis and gallstones. 
Moreover, the study could not identify other significant causes of comet tail artifacts in cholesterolosis. Future studies regarding the relationship between cholesterolosis and the comet tail artifact or RASs are required.

The ultrasonographic diagnoses of gallbladder disease were acute cholecystitis, adenomyomatosis, chronic cholecystitis, cholesterolosis, benign polyp, and gallbladder cancer. Of these diagnoses, adenomyomatosis, cholesterolosis, and benign polyp are not usually included in the indications for cholecystectomy. However, these patients were found to have acute cholecystitis or gallstone on ultrasonography, or biliary colic symptoms, which led to surgery. For this reason, the list of preoperative ultrasonographic diagnoses and the list of clinical indications of cholecystectomy were slightly different. Meanwhile, on preoperative ultrasonographic examination, gallbladder cancer was suspected in five patients. Although the thickness of the lesions was less than $10 \mathrm{~mm}$, they were relatively thick and showed loss of the wall layer. Three of these lesions showed an irregular margin. The final diagnoses of these lesions were adenomyomatosis, chronic cholecystitis, and cholesterolosis. Thus, even if several ultrasonographic findings, including irregular margins and loss of the wall layer, suggest that a lesion is malignant, benignity should be considered in lesions with a comet tail artifact.

In the present study, there were two cases of coexistent chronic cholecystitis and low-grade dysplasia in a thickened gallbladder lesion. The time interval between the gallbladder ultrasonography and cholecystectomy was 14 and 7 days, respectively, which was within the approximately 2-week interval in which no significant changes in the lesion are expected. Nevertheless, because low-grade dysplasia is indicative of a pre-malignant lesion, the possibility that gallbladder lesions would be diagnosed as gallbladder cancer if the time interval is larger cannot be ruled out [24]. In this study, 204 days was the maximum time interval between ultrasonography and cholecystectomy, which may be too short for the development of gallbladder malignancies. Further research investigating longer time intervals in this regard is warranted.

In this study, there were no statistically significant differences in clinical and imaging findings, with the exception of gallstones, among the four diagnoses. The proportion of gallstones in cases of chronic cholecystitis was significantly higher than the corresponding proportion in cases of adenomyomatosis. However, because of the high rates of accompanying gallstones in cases of both chronic cholecystitis (89.2\%) and adenomyomatosis (71.8\%), the use of gallstones to distinguish between these diagnoses is limited. Although cholesterolosis showed a low percentage (33.3\%) of accompanying gallstones and a statistically significant difference compared with chronic cholecystitis, the number of patients diagnosed with cholesterolosis in this study was too small to generalize the result. Thus, gallstones in chronic cholecystitis may be an important associated finding that is often seen; however, it is unreasonable to argue that gallstones may help differentiate other gallbladder diseases. To investigate the proportion of malignant lesions and the ratios among the other benign diseases according to the extent of involvement, this study divided the extent of gallbladder lesions into two categories. However, there were no malignant lesions in either category, and there was no statistically significant difference in the proportion of final pathologic diagnoses between the categories. As a result, it is unlikely that lesion extent can help to differentiate gallbladder lesions with a comet tail artifact. This study compared the length, thickness, and number of comet tail artifacts among the four final pathologic diagnoses, and found no statistically significant differences. Therefore, these clinical and imaging findings, including the shape and number of comet tail artifacts, may not be helpful in differentiating among these four benign diseases.

Meanwhile, there were actually two or more pathologic diagnoses in many patients. However, for convenience of analysis, the final diagnosis was simplified to a single pathologic diagnosis. The pathologic reports did not mention how, or to what extent, the pathologic findings were mixed. However, the reports did contain an indication of which pathologic findings seemed to be more widespread and prominent. The largest or most prominent pathological findings were regarded as the primary pathologic findings, and only the corresponding disease was chosen as the final diagnosis. Thus, simplifying the diagnosis may have led to a limitation in the comparison of the clinical or imaging findings among the 4 diagnoses.

In previous studies, comet tail artifacts were produced by cholesterol crystals and observed in adenomyomatosis of the gallbladder [14,25-27]. However, no study has investigated the relationship between the pathologic diagnosis of gallbladder lesions and the presence of a comet tail artifact. This study is meaningful in that it investigated the pathologic diagnosis of gallbladder lesions with comet tail artifacts on ultrasonographic imaging in a relatively large number of patients, and all cases were benign gallbladder lesions.

This study has a few limitations. First, it was a retrospective study. The pathologic diagnoses were obtained from the whole gallbladder specimens, rather than from the exact lesions corresponding to the comet tail artifacts. As a result, there is a limited one-to-one correspondence between the pathologic diagnosis and imaging findings in the exact area with the comet tail artifact. In addition, whether the portion of the specimen with dysplasia corresponded to the exact area accompanying the comet tail artifact remains unknown. Second, this study simplified the pathologic diagnoses, of 
which two or more could co-exist in each patient, to a single final diagnosis. Third, the study used a single-center design. There were too few cases of cholesterolosis $(n=3)$ and xanthogranulomatous cholecystitis $(n=2)$, which limited the statistical power of the results. Selection bias was also a limitation of this study. This study was conducted only among patients with comet tail artifacts. In future studies, it is necessary to collect consecutive patients regardless of the presence of a comet tail artifact, and to make a direct comparison between the group with the artifacts and the other group without the artifacts. A prospective study with a multicenter design and large cohort is needed in the future.

In conclusion, in this study, all gallbladder lesions with a comet tail artifact on ultrasonographic imaging, regardless of lesion extent and other suspicious ultrasonographic findings such as irregular margin and loss of the wall layer, were benign, including causes of chronic cholecystitis, adenomyomatosis, xanthogranulomatous cholecystitis, and cholesterolosis. Therefore, the comet tail artifact in gallbladder lesions may potentially be a reliable sign suggesting the presence of a benign lesion. Our results may be applicable to the differential diagnosis of gallbladder diseases through gallbladder ultrasonography.

ORCID: Sung Hoon Oh: https://orcid.org/0000-0001-6916-3375; Hyun Young Han: https://orcid.org/0000-0002-9557-3957; Hee Jin Kim: https://orcid.org/0000-0002$6747-664 X$

\section{Conflict of Interest}

No potential conflict of interest relevant to this article was reported.

\section{References}

1. Mellnick VM, Menias CO, Sandrasegaran K, Hara AK, Kielar AZ, Brunt EM, et al. Polypoid lesions of the gallbladder: disease spectrum with pathologic correlation. Radiographics 2015;35:387399.

2. Runner GJ, Corwin MT, Siewert B, Eisenberg RL. Gallbladder wall thickening. AJR Am J Roentgenol 2014;202:W1-W12.

3. van Breda Vriesman AC, Engelbrecht MR, Smithuis RH, Puylaert JB. Diffuse gallbladder wall thickening: differential diagnosis. AJR Am J Roentgenol 2007;188:495-501.

4. Lee KF, Wong J, Li JC, Lai PB. Polypoid lesions of the gallbladder. Am J Surg 2004;188:186-190.

5. Andren-Sandberg A. Diagnosis and management of gallbladder polyps. N Am J Med Sci 2012;4:203-211.

6. Bang SH, Lee JY, Woo H, Joo I, Lee ES, Han JK, et al. Differentiating between adenomyomatosis and gallbladder cancer: revisiting a comparative study of high-resolution ultrasound, multidetector CT, and MR imaging. Korean J Radiol 2014;15:226-234.

7. Ching BH, Yeh BM, Westphalen AC, Joe BN, Qayyum A, Coakley FV. CT differentiation of adenomyomatosis and gallbladder cancer. AJR Am J Roentgenol 2007;189:62-66.

8. Kim SJ, Lee JM, Lee JY, Kim SH, Han JK, Choi Bl, et al. Analysis of enhancement pattern of flat gallbladder wall thickening on MDCT to differentiate gallbladder cancer from cholecystitis. AJR Am J Roentgenol 2008;191:765-771.

9. Yoshimitsu $K$, Honda $H$, Jimi M, Kuroiwa T, Hanada K, Irie $H$, et al. MR diagnosis of adenomyomatosis of the gallbladder and differentiation from gallbladder carcinoma: importance of showing Rokitansky-Aschoff sinuses. AJR Am J Roentgenol 1999;172:15351540.

10. Song ER, Chung WS, Jang HY, Yoon M, Cha EJ. CT differentiation of 1-2-cm gallbladder polyps: benign vs malignant. Abdom Imaging 2014;39:334-341.

11. Feldman MK, Katyal S, Blackwood MS. US artifacts. Radiographics 2009;29:1179-1189.

12. Pattison P, Jeffrey RB Jr, Mindelzun RE, Sommer FG. Sonography of intraabdominal gas collections. AJR Am J Roentgenol 1997;169:1559-1564.

13. Bertrand PB, Levine RA, Isselbacher EM, Vandervoort PM. Fact or artifact in two-dimensional echocardiography: avoiding misdiagnosis and missed diagnosis. J Am Soc Echocardiogr 2016;29:381-391.

14. Lafortune M, Gariepy G, Dumont A, Breton G, Lapointe R. The V-shaped artifact of the gallbladder wall. AJR Am J Roentgenol 1986;147:505-508.

15. Bonatti M, Vezzali N, Lombardo F, Ferro F, Zamboni G, Tauber M, et al. Gallbladder adenomyomatosis: imaging findings, tricks and pitfalls. Insights Imaging 2017:8:243-253.

16. Kumar V, Abbas AK, Aster JC. Robbins basic pathology. 10th ed. Philadelphia, PA: Elsevier, 2018.

17. Goldblum JR, Lamps LW, McKenney JK, Myers JL. Rosai and Ackerman's surgical pathology. 11th ed. Cambridge, MA: Elsevier, 2018.

18. Singh VP, Rajesh S, Bihari C, Desai SN, Pargewar SS, Arora A. Xanthogranulomatous cholecystitis: what every radiologist should know. World J Radiol 2016;8:183-191.

19. Smith EA, Dillman JR, Elsayes KM, Menias CO, Bude RO. Crosssectional imaging of acute and chronic gallbladder inflammatory disease. AJR Am J Roentgenol 2009;192:188-196.

20. Dattal DS, Kaushik R, Gulati A, Sharma VK. Morphological spectrum of gall bladder lesions and their correlation with cholelithiasis. Int J Res Med Sci 2017:5:840-846.

21. Carey MC. Pathogenesis of gallstones. Am J Surg 1993;165:410419.

22. Van Erpecum KJ. Pathogenesis of cholesterol and pigment gallstones: an update. Clin Res Hepatol Gastroenterol 
2011:35:281-287.

23. Sandri L, Colecchia A, Larocca A, Vestito A, Capodicasa S, Azzaroli $F$, et al. Gallbladder cholesterol polyps and cholesterolosis. Minerva Gastroenterol Dietol 2003;49:217-224.

24. Katabi N. Neoplasia of gallbladder and biliary epithelium. Arch Pathol Lab Med 2010;134:1621-1627.

25. Shapiro RS, Winsberg F. Comet-tail artifact from cholesterol crystals: observations in the postlithotripsy gallbladder and an in vitro model. Radiology 1990;177:153-156.

26. Hwang Jl, Chou YH, Tsay SH, Chiang JH, Chang CY, Boland GW, et al. Radiologic and pathologic correlation of adenomyomatosis of the gallbladder. Abdom Imaging 1998;23:73-77.

27. Raghavendra BN, Subramanyam BR, Balthazar EJ, Horii SC, Megibow AJ, Hilton S. Sonography of adenomyomatosis of the gallbladder: radiologic-pathologic correlation. Radiology 1983;146:747-752. 